\title{
Sigmoid Sinus Diverticulum, Dehiscence, and Venous Sinus Stenosis: Potential Causes of Pulsatile Tinnitus in Patients with Idiopathic Intracranial Hypertension?
}

\author{
(D).A. Lansley, (DW. Tucker, (DM.R. Eriksen, DP. Riordan-Eva, and (DS.E.J. Connor
}

\begin{abstract}
BACKGROUND AND PURPOSE: Pulsatile tinnitus is experienced by most patients with idiopathic intracranial hypertension. The pathophysiology remains uncertain; however, transverse sinus stenosis and sigmoid sinus diverticulum/dehiscence have been proposed as potential etiologies. We aimed to determine whether the prevalence of transverse sinus stenosis and sigmoid sinus diverticulum/ dehiscence was increased in patients with idiopathic intracranial hypertension and pulsatile tinnitus relative to those without pulsatile tinnitus and a control group.
\end{abstract}

MATERIALS AND METHODS: CT vascular studies of patients with idiopathic intracranial hypertension with pulsatile tinnitus ( $n=42$ ), without pulsatile tinnitus $(n=37)$, and controls $(n=75)$ were independently reviewed for the presence of severe transverse sinus stenosis and sigmoid sinus diverticulum/dehiscence according to published criteria. The prevalence of transverse sinus stenosis and sigmoid sinus diverticulum/dehiscence in patients with idiopathic intracranial hypertension with pulsatile tinnitus was compared with that in the nonpulsatile tinnitus idiopathic intracranial hypertension group and the control group. Further comparisons included differing degrees of transverse sinus stenosis (50\% and $75 \%$ ), laterality of transverse sinus stenosis/sigmoid sinus diverticulum/dehiscence, and ipsilateral transverse sinus stenosis combined with sigmoid sinus diverticulum/dehiscence.

RESULTS: Severe bilateral transverse sinus stenoses were more frequent in patients with idiopathic intracranial hypertension than in controls $(P<.001)$, but there was no significant association between transverse sinus stenosis and pulsatile tinnitus within the idiopathic intracranial hypertension group. Sigmoid sinus dehiscence (right- or left-sided) was also more common in patients with idiopathic intracranial hypertension compared with controls $(P=.01)$, but there was no significant association with pulsatile tinnitus within the idiopathic intracranial hypertension group.

CONCLUSIONS: While our data corroborate previous studies demonstrating increased prevalence of sigmoid sinus diverticulum/dehiscence and transverse sinus stenosis in idiopathic intracranial hypertension, we did not establish an increased prevalence in patients with idiopathic intracranial hypertension with pulsatile tinnitus compared with those without. It is therefore unlikely that these entities represent a direct structural correlate of pulsatile tinnitus in patients with idiopathic intracranial hypertension.

ABBREVIATIONS: $I \mathrm{IH}=$ idiopathic intracranial hypertension; PT = pulsatile tinnitus; SSDD = sigmoid sinus diverticulum/dehiscence; TSS = transverse sinus stenosis

$\mathbf{P}$ ulsatile tinnitus (PT) is the perception of an abnormal sound that is synchronous with the heartbeat. The phenomenon may result from abnormal mechanical somatosound production

Received September 22, 2016; accepted after revision April 29, 2017.

From the Barts Health National Health Service Trust (J.A.L.), London, UK; King's College Hospital (W.T., M.R.E., P.R.-E., S.E.J.C.), Denmark Hill, London, UK; Aleris Roentgen Institutte Stavanger (M.R.E.), Stavanger, Norway; and Guy's and St Thomas' Hospital (S.E.J.C.), London, UK.

Paper previously presented at: Annual Meeting and Refresher Course of the European Society of Head and Neck Radiology, September 22-24, 2016; Leiden, the Netherlands.

Please address correspondence to Joseph A. Lansley, MBBS, Neuroradiology Department, Royal London Hospital, Whitechapel Rd, London, El 1BB, UK; e-mail:

dr.joseph.lansley@gmail.com

http://dx.doi.org/10.3174/ajnr.A5277

(due to abnormal turbulence or location of vascular flow) or enhanced perception (due to increased transmission of the somatosound or reduced conduction of normal sound). ${ }^{1} \mathrm{PT}$ is a wellrecognized feature of idiopathic intracranial hypertension (IIH), being present in approximately $60 \%$ of patients. ${ }^{2-4}$ The pathophysiology of PT in these patients is unknown; however, observed variations in transverse and sigmoid sinus anatomy seen in IIH have been proposed as a possible underlying cause.

Transverse sinus stenosis (TSS) is well-described as a cause of $\mathrm{PT}$, and resolution of symptoms has been reported following successful venous sinus stent placement. ${ }^{5-8}$ Venous sinus stent placement performed as a treatment for IIH has also resulted in resolution of PT in affected patients, ${ }^{7,8}$ which lends support to the 

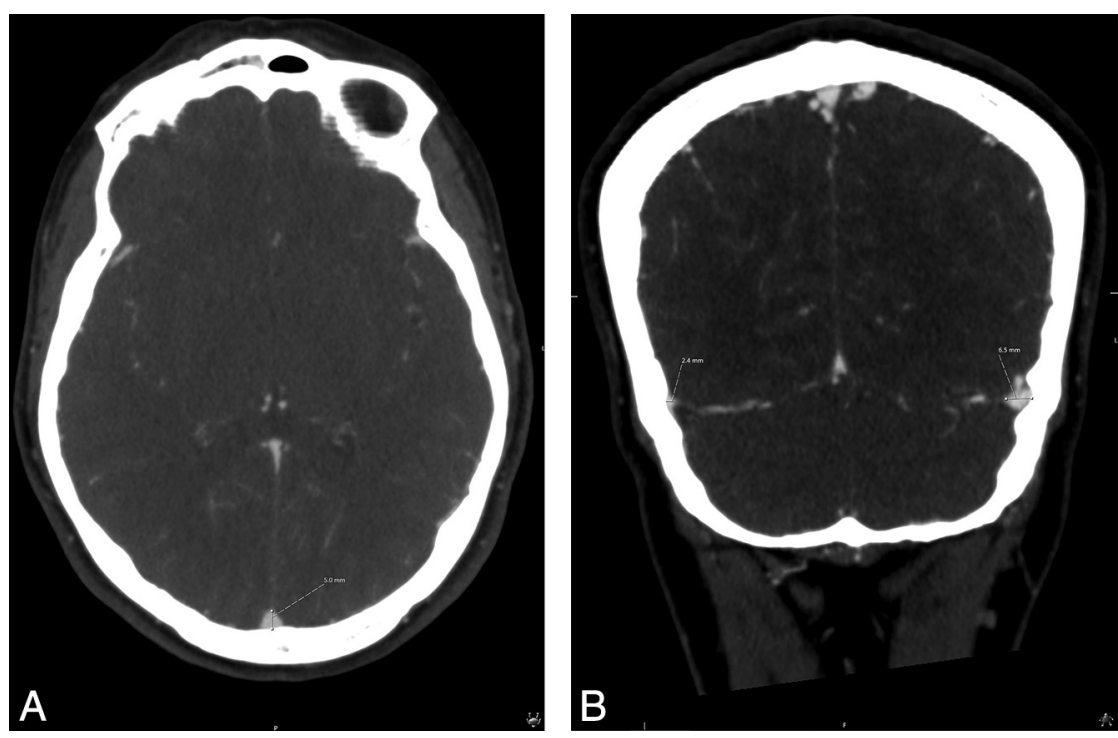

FIG 1. A, Superior sagittal sinus height measurement $(2 \mathrm{~cm}$ above the torcula). $B$, Transverse sinus stenosis measurement.

proposal that the resulting turbulent venous flow may be the somatosound responsible for PT in IIH. ${ }^{9,10}$ However, PT is not seen in all patients with IIH with TSS, and other factors may be required for the symptom to manifest.

More recently, sigmoid sinus diverticulum and/or dehiscence (SSDD) has been shown to be associated with PT. ${ }^{1,11-16}$ This association has been noted to be particularly frequent in patients with elevated body mass indices and those with imaging features of IIH, ${ }^{1,14,15}$ leading some authors to suggest that SSDD may reflect a manifestation of subclinical IIH. ${ }^{2,14}$

Interventional studies have revealed that endovascular stenting of TSS or surgical correction of SSDD may resolve $\mathrm{PT}^{5,7,8,13,17-19}$; however, these procedures are not without complications. ${ }^{12}$ Understanding the underlying mechanism is therefore essential to enable appropriate targeting of therapy.

We aimed to determine whether the prevalence of TSS and SSDD was increased in patients with IIH with PT relative to patients with IIH without PT and a control group without IIH.

\section{MATERIALS AND METHODS \\ Subjects}

One hundred seventy consecutive patients diagnosed with IIH were identified from a clinical data base at our institution and selected for retrospective review. IIH diagnosis was made with the support of CSF pressure measurements and according to the modified Dandy criteria ${ }^{20}$ in all except 1 patient in whom lumbar puncture was precluded due to cerebellar tonsillar herniation. Case notes were reviewed for demographic and clinical details, including a history of pulsatile tinnitus. Laterality of PT was not consistently recorded and hence not documented. Patients were excluded if there was no CT venogram available for evaluation. When $>1$ CT venogram had been obtained on the same patient, the examination closest to the diagnosis of IIH was selected for analysis. Seventy-nine patients with IIH (mean, $32 \pm 5.8$ years of age; male/female ratio, 4:75) were available for evaluation.
A cohort of control subjects was generated by using contemporaneous CT angiograms obtained on the same CT scanner for reasons other than suspected venous pathology or pulsatile tinnitus. A list of CTA examinations was generated by searching the PACS archive for intracranial CTAs performed in the study period (January 1, 2005, to May 22, 2015) that contained the text "normal" in the body of the report. The resulting 1250 studies were ranked by age with subjects younger than 16 and older than 45 years of age excluded to closely model the IIH cohort. The report text of the remaining scans was manually screened, and studies with abnormal findings were excluded. The resulting 183 CTAs were reviewed, and further exclusions were made if there was confounding pathology or if venous opacification was insufficient (defined as $<160 \mathrm{HU}$ measured at the torcula). After this process, 75 control subjects were available for evaluation.

The study was reviewed by the local National Health Service Research Ethics Committee and was considered to represent "service evaluation."

\section{Radiologic Assessment}

CTAs and CT venograms were obtained with a LightSpeed 16 scanner (GE Healthcare, Milwaukee, Wisconsin). Scanning parameters were 0.625-mm collimation; pitch, 0.938:1; noise index, 2.2; automilliampere limit, 380 .

The presence of venous sinus stenosis, sigmoid plate dehiscence, and diverticula was assessed by 2 neuroradiologists independently (J.A.L. and M.R.E., with 9 and 15 years of radiology experience, respectively). Venous sinus luminal dimensions were first evaluated with maximum-intensity-projection reconstructions to provide an overview of the narrowest portion of the sinus encountered from the torcula to the distal sigmoid sinus. Multiplanar reformats $(0.6 \mathrm{~mm}$ thick) at standard magnifications and perpendicular to the plane of the skull vault were then used to assess the height of the sinuses. Percentage stenoses were calculated as the ratio of the maximal narrowing relative to the maximal height of the superior sagittal sinus (measured $2 \mathrm{~cm}$ above the torcula, Fig 1). Stenoses were categorized as $>50 \%$ and $>75 \%$ relative to the superior sagittal sinus according to previously published criteria. ${ }^{21}$

A rigorous, objective radiographic classification of SSDDs is yet to be established. For this study, we adapted a previously described methodology, ${ }^{1}$ using MPR reconstructions and an edgesharpening tool available on our PACS. Assessments were made with standard bone windows (width, $4000 \mathrm{HU}$ centered at 400 $\mathrm{HU})$. Dehiscence was characterized as complete absence of cortical bone adjacent to the sigmoid sinus (air-on-sinus sign) evident on at least 2 consecutive 0.6 - $\mathrm{mm}$ sections in 2 orthogonal planes (Fig 2). "Sigmoid sinus diverticulum" was defined as a focal outpouching of the normal semicircular sigmoid sinus groove ex- 


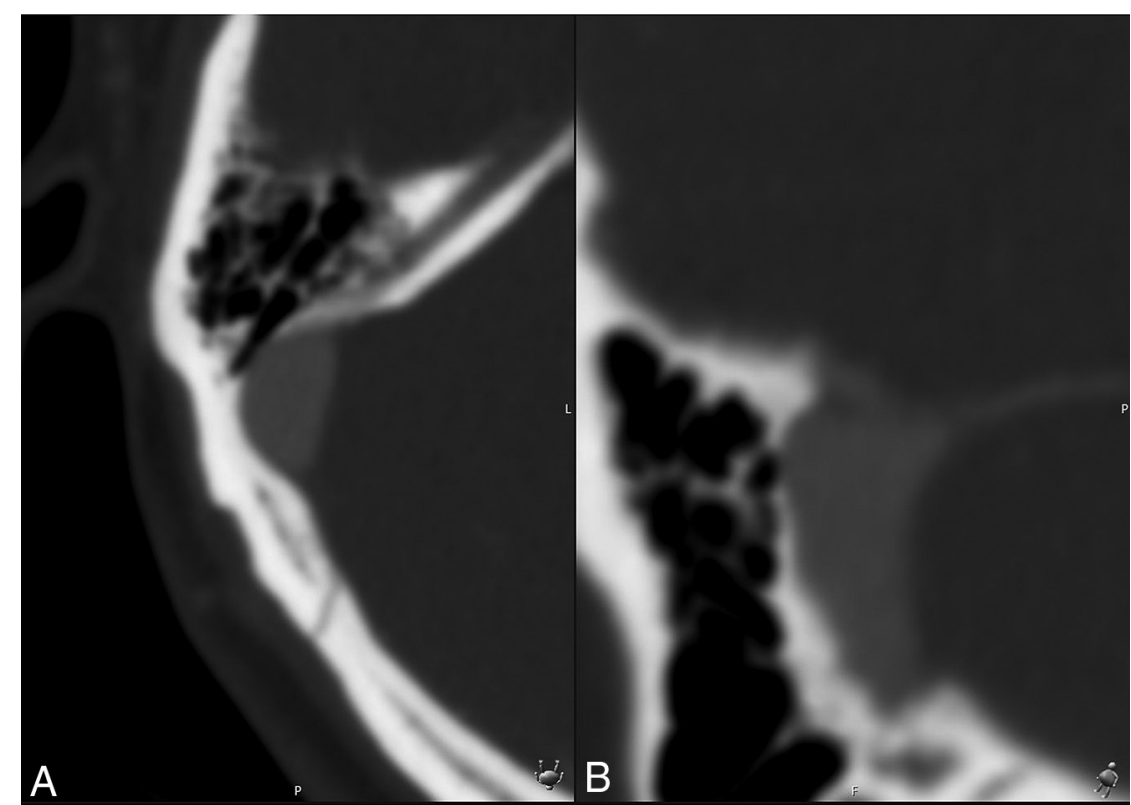

FIG 2. Dehiscence of the sigmoid sinus wall: "air-on-sinus" sign in the axial $(A)$ and sagittal planes (B).

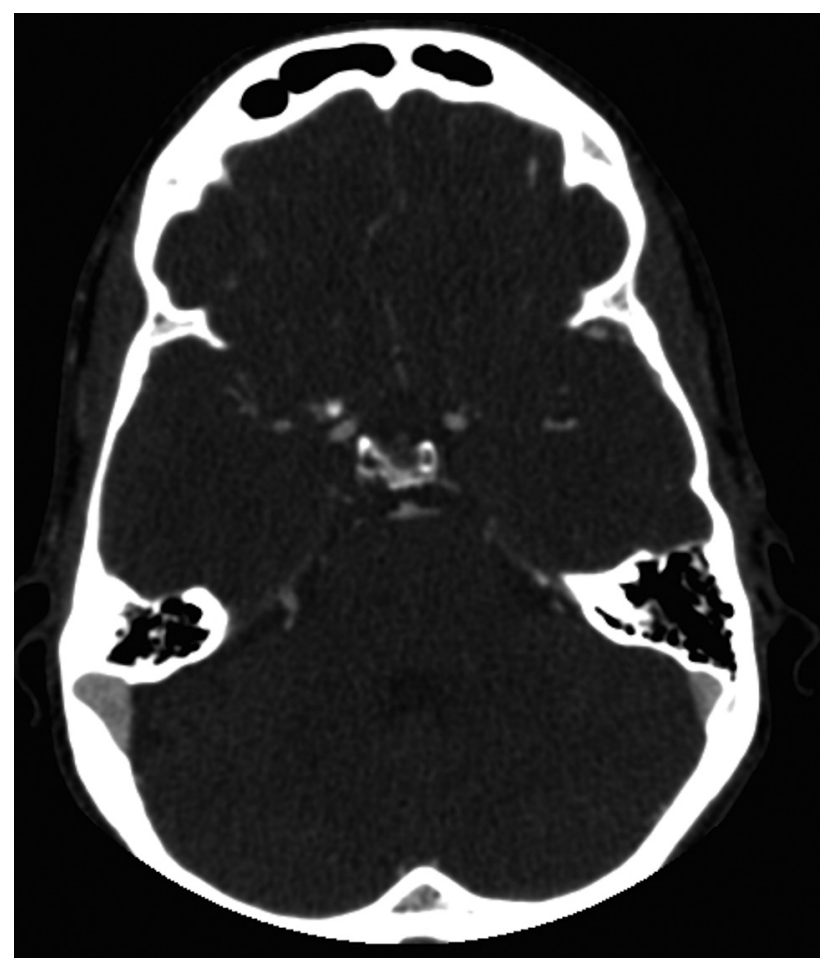

FIG 3. Example of a right sigmoid diverticulum.

panding into the mastoid air cells and/or temporal bone cortex (Fig 3). ${ }^{18}$

Consensus review was undertaken for all discrepant cases derived from the initial independent readings.

\section{Statistical Analysis}

Data were entered into an Excel spreadsheet (Excel for Mac, 2011; Microsoft, Redmond, Washington). The statistical package StatPlus (AnalystSoft, Walnut, California) was used for descriptive statistics.
The prevalence of TSS $(>75 \%$ and $>50 \%$ ), sigmoid sinus dehiscence, and sigmoid sinus diverticulum in patients with IIH was compared with that in the control group to see whether the previously reported associations were replicated in our cohort. Further subgroup analyses were performed to compare the PT-IIH group with the controls and the non-PT IIH group. Group comparisons were also performed to compare the prevalence of ipsilateral TSS and SSDD in the cohorts in case simultaneous abnormalities were needed for PT to manifest. Statistical comparison was performed with $\chi^{2}$ or Fisher exact tests as appropriate, with $P<.05$ being required for significance.

We performed further exploratory between-group comparisons, taking into account variations in the laterality of TSS and SSDD (ie, left, right, unilateral, bilateral, and either unilateral or bilateral).

\section{RESULTS}

There was no significant difference in the average age of the IIH group ( $32 \pm 5.8$ years) and control group ( $32 \pm 10.2$ years). Of the 79 patients with IIH, 42 had PT. The average body mass index in the IIH group was 37, and there was no significant difference in body mass indices between patients with IIH with and without PT. The IIH group was almost exclusively female (75:4), whereas the control group comprised 35 men and 40 women.

The characteristics and imaging analysis results of the groups are summarized in the Table.

Patients with IIH were significantly more likely to have a severe grade of venous sinus stenosis ( $>50 \%$ and $>75 \%$ ) compared with controls. When both left and right transverse sigmoid sinus drainage pathways were considered together, $49 \%$ of patients with IIH had $>75 \%$ stenosis affecting 1 side compared with just $7 \%$ of controls $(P<.001)$. This significance was maintained for the PTIIH subgroup analysis, but there was no significant association between severe dural venous sinus stenosis and PT within the IIH group.

Dehiscence, as defined by the "air-on-sinus" sign, was more common on the right in all groups. In the IIH group, $41 \%$ of all temporal bones were dehiscent, compared with $27 \%$ of controls $(P=.01)$. The significance of this difference was retained when controls were compared with the PT-IIH subgroup, but there was no significant difference between patients with and without PT in the IIH group.

Sigmoid sinus diverticula were twice as common in patients with IIH compared with controls ( $8 \%$ versus $4 \%$ ); however, the difference was not significant, and no other significant differences were found in the subgroup analyses.

There was no significant difference in the prevalence of ipsi- 


\begin{tabular}{|c|c|c|c|c|c|c|c|}
\hline & $\begin{array}{c}\text { Subgroup } 1 \\
\text { (IIH with PT) } \\
\text { (n= 42) }\end{array}$ & $\begin{array}{c}\text { Subgroup } 2 \\
\text { (IIH without PT) } \\
(n=37)\end{array}$ & $\begin{array}{l}\text { Patients with } \\
\text { IIH (Combined) } \\
(n=79)\end{array}$ & $\begin{array}{c}\text { Controls } \\
(n=75)\end{array}$ & $P$ & $P 1$ & P2 \\
\hline Rt stenosis $>75 \%$ & $21(50 \%)$ & $14(38 \%)$ & $35(44 \%)$ & $3(4 \%)$ & .28 & $<.001^{\mathrm{a}}$ & $<.001^{\circ}$ \\
\hline Lt stenosis $>75 \%$ & $21(50 \%)$ & $21(57 \%)$ & $42(53 \%)$ & $7(9 \%)$ & .55 & $<.001^{\mathrm{a}}$ & $<.001^{\mathrm{a}}$ \\
\hline Rt or Lt stenosis $>75 \%$ & $42(50 \%)$ & $35(47 \%)$ & $77(49 \%)$ & $10(7 \%)$ & .73 & $<.001$ & $<.001^{\circ}$ \\
\hline Unilateral $>75 \%$ stenosis & $16(38 \%)$ & $15(41 \%)$ & $33(42 \%)$ & $10(13 \%)$ & .82 & $.002^{\mathrm{a}}$ & $<.001^{\circ}$ \\
\hline Bilateral $>75 \%$ stenoses & $13(31 \%)$ & $9(24 \%)$ & $22(28 \%)$ & 0 & .51 & $<.001^{\mathrm{a}}$ & $<.001^{\circ}$ \\
\hline Unilateral or bilateral $>75 \%$ stenosis & $29(69 \%)$ & $24(65 \%)$ & $55(70 \%)$ & $10(13 \%)$ & 69 & $<.001^{\mathrm{a}}$ & $<.001^{\circ}$ \\
\hline Rt stenosis $>50 \%$ & $30(71 \%)$ & $28(76 \%)$ & $58(73 \%)$ & $6(8 \%)$ & .67 & $<.001^{\mathrm{a}}$ & $<.001^{\circ}$ \\
\hline Lt stenosis $>50 \%$ & $33(78 \%)$ & $32(86 \%)$ & $65(82 \%)$ & $19(25 \%)$ & .36 & $<.001^{\mathrm{a}}$ & $<.001^{\circ}$ \\
\hline Rt or Lt stenosis $>50 \%$ & $63(75 \%)$ & $60(81 \%)$ & $123(78 \%)$ & $25(16 \%)$ & .36 & $<.001^{\mathrm{a}}$ & $<.001^{\circ}$ \\
\hline Unilateral $>50 \%$ stenosis & $7(17 \%)$ & $10(27 \%)$ & $17(22 \%)$ & $21(28 \%)$ & .26 & .17 & .35 \\
\hline Bilateral $>50 \%$ stenoses & $28(67 \%)$ & $25(68 \%)$ & $53(67 \%)$ & $2(3 \%)$ & .93 & $<.001^{\mathrm{a}}$ & $<.001^{\circ}$ \\
\hline Unilateral or bilateral $>50 \%$ stenoses & $35(83 \%)$ & $35(94 \%)$ & $70(89 \%)$ & $23(31 \%)$ & .12 & $<.001^{\mathrm{a}}$ & $<.001^{\circ}$ \\
\hline Rt diverticulum & $5(12 \%)$ & $3(8 \%)$ & $8(10 \%)$ & $6(8 \%)$ & .72 & .48 & .65 \\
\hline Lt diverticulum & $2(5 \%)$ & $2(5 \%)$ & $4(5 \%)$ & 0 & 1 & .13 & .12 \\
\hline Rt or Lt diverticulum & $7(8 \%)$ & $5(7 \%)$ & $12(8 \%)$ & $6(4 \%)$ & .71 & .17 & .18 \\
\hline Unilateral diverticulum & $5(12 \%)$ & $5(14 \%)$ & $10(13 \%)$ & $6(8 \%)$ & .83 & .49 & .34 \\
\hline Bilateral diverticula & $1(2 \%)$ & 0 & $1(1 \%)$ & 0 & 1 & .36 & 1 \\
\hline Unilateral or bilateral diverticula & $6(14 \%)$ & $5(14 \%)$ & $11(14 \%)$ & $6(8 \%)$ & .92 & .28 & .24 \\
\hline Rt dehiscence & $24(57 \%)$ & $16(43 \%)$ & $40(51 \%)$ & $28(37 \%)$ & .22 & $.04^{\mathrm{a}}$ & .10 \\
\hline Lt dehiscence & $12(29 \%)$ & $12(32 \%)$ & $24(30 \%)$ & $12(16 \%)$ & .71 & .11 & $.04^{\mathrm{a}}$ \\
\hline Rt or Lt dehiscence & $36(42 \%)$ & $28(38 \%)$ & $64(41 \%)$ & $40(27 \%)$ & .52 & $.01^{\mathrm{a}}$ & $.01^{\mathrm{a}}$ \\
\hline Unilateral dehiscence & $18(43 \%)$ & $12(32 \%)$ & $30(38 \%)$ & $26(35 \%)$ & .34 & .38 & .70 \\
\hline Bilateral dehiscence & $9(21 \%)$ & $8(22 \%)$ & $17(22 \%)$ & $7(9 \%)$ & .98 & .07 & $.04^{\mathrm{a}}$ \\
\hline Unilateral or bilateral dehiscence & $27(64 \%)$ & $20(54 \%)$ & $47(60 \%)$ & $33(44 \%)$ & .36 & $.04^{\mathrm{a}}$ & $.05^{\mathrm{a}}$ \\
\hline
\end{tabular}

Note:- P indicates IIH with PT vs IIH without PT; PI, IIH with PT vs controls; P2, IIH (combined) vs controls; Rt, right; Lt, left.

a Significant.

lateral stenosis and dehiscence or ipsilateral stenosis and diverticulum between IIH patients with PT and those without PT.

\section{DISCUSSION}

Venous tinnitus in IIH has been determined to be the most frequent cause of PT in some series, ${ }^{4}$ and it is proposed that this may have a direct anatomic correlate with either TSS or SSDD. An increased prevalence of TSS and SSDD has been noted in patients with $\mathrm{PT},{ }^{12,22}$ and the symptoms of PT have responded to previous targeted treatments to the transverse sigmoid sinus in patients with and without IIH. , $7,8,17-19^{-19}$

Although both TSS and SSDD have been linked to IIH, to the best of our knowledge, no previous studies have investigated the frequency in patients with $\mathrm{IIH}$ with and without PT. We aimed to evaluate whether the prevalence of either entity could explain the occurrence of PT in a cohort of patients with confirmed IIH. Alternatively, TSS and SSDD may have a noncausal association with PT and CSF, or brain pulsation may be transmitted to the inner ear structures by alternative mechanisms. ${ }^{22,23}$

TSS is present in most patients with $\mathrm{IIH}^{21,24,25}$; however, the etiology remains controversial. Pre-existing areas of TSS may be implicated in the development of $\mathrm{IIH}^{7,26}$; however, it is also postulated that they occur secondary to raised CSF pressure, and reversibility of stenoses has been demonstrated by lowering CSF pressure. ${ }^{27}$ Patients with TSS secondary to IIH may experience PT due to turbulent venous flow directly related to the venous stenoses or due to periodic narrowing caused by arterial pulsations transmitted to the venous sinuses across the CSF. ${ }^{28}$

Patients with PT and SSDD have been found to have clinical and imaging characteristics of IIH. ${ }^{1,12,15}$ A number of mechanisms have been proposed to explain this association. It is possible that the impact of pulsatile, turbulent blood flow on the sinus wall could cause the formation of SSDD. ${ }^{15,18}$ Alternatively the presence of SSDD may be a direct result of raised CSF pressures. ${ }^{12,16,29}$ It has also been postulated that sigmoid sinus dehiscence could cause PT in these patients due to increased transmission of turbulent flow to the inner ear after loss of the insulating properties afforded by cortical bone. ${ }^{1,16,30}$ Although our findings did demonstrate an association between dehiscence and IIH, there was no association within the IIH group to support a direct causal relationship. We also investigated the suggestion that a combination of TSS and SSDD may be required to cause PT, ${ }^{1}$ but we found no increased frequency of ipsilateral TSS and SSDD in our PT cohort with IIH to support this.

It is possible that dehiscence involving specific locations on the curve of the sigmoid sinus results in PT symptoms. ${ }^{13}$ We did not categorize dehiscence in this way; this choice could have reduced our ability to detect a significant association. We also found that tiny focal areas of dehiscence were commonly present, even in control subjects, which casts some doubt about their clinical significance.

We did not distinguish different types of stenosis: tapered "extraluminal” narrowing versus "intraluminal" narrowing typified by arachnoid granulations. ${ }^{21}$ This could also have limited our ability to detect a significant association between stenosis and PT.

It is unclear whether sigmoid sinus diverticula and dehiscence represent separate entities or whether there is an underlying pathophysiologic spectrum of disease. ${ }^{1,15}$ Diverticula may cause turbulent venous flow and lead to symptomatic PT when coexisting with elevated intracranial pressure, independent of TSS; how- 
ever, our IIH cohort did not reveal any association between PT and diverticula to lend support to this theory.

We explored the combined effect of TSS and ipsilateral SSDD, but it is possible that a more complex combination of anatomic features is required to cause PT or that the pathophysiology differs between patients. It has been shown that ipsilateral venous outflow dominance, high jugular bulb, and temporal bone hyperpneumatization may also be important in the manifestation of SSDD as PT. ${ }^{13,16}$ Complex interactions with venous dominance, the site and size of dehiscence, and the type of stenosis (intraluminal versus extraluminal) were not fully evaluated in our study. Although we did not document venous dominance directly, diverticula and dehiscence were observed more frequently on the right; this feature is of interest because this is more likely to be the dominant sinus. ${ }^{31}$

Our findings reveal a high prevalence of $\mathrm{PT}$ in $\mathrm{IIH}$, which is in line with previous studies. ${ }^{3}$ The data also corroborate previous research indicating a high frequency of transverse sinus stenosis and sigmoid sinus wall abnormalities in IIH. However, we found no significant association between PT and venous sinus or sinus wall abnormalities within the IIH group, suggesting that a direct causal link is unlikely. Furthermore, ipsilateral dehiscence and TSS abnormalities were not significantly more frequent in the PT group; this finding suggests that even when combined, these factors are not sufficient to cause PT.

Our dataset contained a large validated cohort of patients diagnosed with IIH, corroborated by CSF pressure measurements; however, the study design has some important limitations. First, the retrospective nature meant that there was nonuniformity of data. Most important, we had limited information on the laterality of PT. It is recognized that PT in IIH is most commonly unilateral, ${ }^{32}$ and this information would have allowed a greater confidence in relating PT to the venous correlates. The lack of well-defined criteria and the subjective nature of the scoring for SSDD are also potential drawbacks; however, these were similarly applied across groups with standardized viewing parameters, and a consensus review was undertaken in cases of disagreement.

Another potential bias was introduced by including only patients with IIH who had undergone CT venography, which a large number of patients in the data base had not undertaken. The use of CTAs for the control group meant that observer blinding was not possible, and cases could be distinguished from controls. Nevertheless, observers were blinded to the presence of PT within the IIH group, and the question of this association was the primary aim of this study.

No previous studies describe the incidence of SSDD/TSS in confirmed IIH cases, to our knowledge; therefore, we were unable to perform a power calculation, and the sample size was determined by the size of the clinical data base.

Finally, the control group was not matched for sex, resulting in a female bias for the IIH cohort. Previous control cohorts have comprised subjects with middle ear symptoms referred for ear, nose, and throat investigations. The relatively high proportion of SSDD abnormalities found in these subjects (up to 18\%) may misrepresent the general population. ${ }^{14}$ We thought it was important to know the prevalence of SSDD in the general population to better understand the likelihood of SSDD as a coincidental find- ing. A mixed-sex control group was therefore used to provide data about the incidence of SSDD.

Four percent of controls in our cohort had a combined conduit score of $<5$, in line with the $7 \%$ of controls in the study by Farb et al. ${ }^{21}$ Sigmoid sinus dehiscence was more common in our control cohort ( $44 \%$ of patients, $53 \%$ of ears) compared with $1.2 \%$ and $18 \%$ of incidental SSDD reported in previous studies. ${ }^{12,14}$ This discrepancy is likely to reflect shortcomings of CT assessment. Only tiny focal areas of apparent bone loss were required to be categorized as dehiscence; however, as previously shown in temporal bone histologic studies, bone thickness of $<0.1 \mathrm{~mm}$ can be falsely read as dehiscent. ${ }^{33,34}$ We also found a higher incidence of sigmoid sinus diverticula in our control cohort than described in previous studies: $8 \%$ compared with $0.5 \%$ reported by Grewal et al, ${ }^{14}$ which may reflect differences in the demographic studied or possibly be due to sampling bias inherent in our smaller sample size.

\section{CONCLUSIONS}

Although TSS and sigmoid sinus dehiscence were shown to be more frequently present in patients with IIH compared with controls, our findings do not support the proposed pathophysiologic mechanism that SSDD or TSS are directly responsible for PT in IIH. The finding of SSDD in a patient with PT may be coincidental, and patients considering surgical intervention should be counseled accordingly.

Disclosures: Marianna R. Eriksen—UNRELATED: Employment: Aleris Roentgen Institut Stavanger, Norway.

\section{REFERENCES}

1. Harvey RS, Hertzano R, Kelman SE, et al. Pulse-synchronous tinnitus and sigmoid sinus wall anomalies: descriptive epidemiology and the idiopathic intracranial hypertension patient population. Otol Neurotol 2014;35:7-15 CrossRef Medline

2. Shaw GY, Million SK. Benign intracranial hypertension: a diagnostic dilemma. Case Rep Otolaryngol 2012;2012:814696 CrossRef Medline

3. Markey KA, Mollan SP, Jensen RH, et al. Understanding idiopathic intracranial hypertension: mechanisms, management, and future directions. Lancet Neurol 2016;15:78-91 CrossRef Medline

4. Sismanis A. Pulsatile tinnitus: a 15-year experience. Am J Otol 1998; 19:472-77 Medline

5. Baomin L, Yongbing S, Xiangyu C. Angioplasty and stenting for intractable pulsatile tinnitus caused by dural venous sinus stenosis: a case series report. Otol Neurotol 2014;35:366-70 CrossRef Medline

6. Russell EJ, De Michaelis BJ, Wiet R, et al. Objective pulse-synchronous "essential" tinnitus due to narrowing of the transverse dural venous sinus. Int Tinnitus J 1995;1:127-37 Medline

7. Donnet A, Metellus P, Levrier O, et al. Endovascular treatment of idiopathic intracranial hypertension: clinical and radiologic outcome of 10 consecutive patients. Neurology 2008;70:641-47 CrossRef Medline

8. Ahmed RM, Wilkinson M, Parker GD, et al. Transverse sinus stenting for idiopathic intracranial hypertension: a review of 52 patients and of model predictions. AJNR Am J Neuroradiol 2011;32:1408-14 CrossRef Medline

9. Sismanis A. Pulsatile tinnitus: contemporary assessment and management. Curr Opin Otolaryngol Head Neck Surg 2011;19:348-57 CrossRef Medline

10. Jindal M, Hiam L, Raman A, et al. Idiopathic intracranial hypertension in otolaryngology. Eur Arch Otorhinolaryngol 2009;266:803-06 CrossRef Medline 
11. Mattox DE, Hudgins P. Algorithm for evaluation of pulsatile tinnitus. Acta Otolaryngol 2008;128:427-31 CrossRef Medline

12. Schoeff S, Nicholas B, Mukherjee S, et al. Imaging prevalence of sigmoid sinus dehiscence among patients with and without pulsatile tinnitus. Otolaryngol Head Neck Surg 2014;150:841-46 CrossRef Medline

13. Geng W, Liu Z, Fan Z. CT characteristics of dehiscent sigmoid plates presenting as pulsatile tinnitus: a study of 23 patients. Acta Radiol 2015;56:1404-08 CrossRef Medline

14. Grewal AK, Kim HY, Comstock RH 3rd, et al. Clinical presentation and imaging findings in patients with pulsatile tinnitus and sigmoid sinus diverticulum/dehiscence. Otol Neurotol 2014;35:16-21 CrossRef Medline

15. Liu Z, Dong C, Wang X, et al. Association between idiopathic intracranial hypertension and sigmoid sinus dehiscence/diverticulum with pulsatile tinnitus: a retrospective imaging study. Neuroradiology 2015;57:747-53 CrossRef Medline

16. Zhao P, Lv H, Dong C, et al. CT evaluation of sigmoid plate dehiscence causing pulsatile tinnitus. Eur Radiol 2016;26:9-14 CrossRef Medline

17. Sanchez TG, Murao M, de Medeiros IR, et al. A new therapeutic procedure for treatment of objective venous pulsatile tinnitus. Int Tinnitus J 2002;8:54-57 Medline

18. Eisenman DJ. Sinus wall reconstruction for sigmoid sinus diverticulum and dehiscence: a standardized surgical procedure for a range of radiographic findings. Otol Neurotol 2011;32:1116-19 CrossRef Medline

19. Otto KJ, Hudgins PA, Abdelkafy W, et al. Sigmoid sinus diverticulum: a new surgical approach to the correction of pulsatile tinnitus. Otol Neurotol 2007;28:48-53 CrossRef Medline

20. Friedman DI, Jacobson DM. Diagnostic criteria for idiopathic intracranial hypertension. Neurology 2002;59:1492-95 CrossRef Medline

21. Farb RI, Vanek I, Scott JN, et al. Idiopathic intracranial hypertension: the prevalence and morphology of sinovenous stenosis. Neurology 2003;60:1418-24 CrossRef Medline

22. Chiarella G, Bono F, Cassandro C, et al. Bilateral transverse sinus stenosis in patients with tinnitus. Acta Otorhinolaryngol Ital 2012;32: $238-43$ Medline
23. Shulman A. Brain pulsatility and pulsatile tinnitus: clinical types. Int Tinnitus J 2012;17:2-3 Medline

24. Higgins JN, Gillard JH, Owler BK, et al. MR venography in idiopathic intracranial hypertension: unappreciated and misunderstood. J Neurol Neurosurg Psychiatry 2004;75:621-25 CrossRef Medline

25. Johnston I, Kollar C, Dunkley S, et al. Cranial venous outflow obstruction in the pseudotumour syndrome: incidence, nature and relevance. J Clin Neurosci 2002;9:273-78 CrossRef Medline

26. Higgins JN, Owler BK, Cousins C, et al. Venous sinus stenting for refractory benign intracranial hypertension. Lancet 2002;359: 228-30 CrossRef Medline

27. Scoffings DJ, Pickard JD, Higgins JN. Resolution of transverse sinus stenoses immediately after CSF withdrawal in idiopathic intracranial hypertension. J Neurol Neurosurg Psychiatry 2007;78:911-12 CrossRef Medline

28. Sismanis A. Otologic manifestations of benign intracranial hypertension syndrome: diagnosis and management. Laryngoscope 1987; 97(8 pt 2 suppl 42):1-17 Medline

29. Butros SR, Goncalves LF, Thompson D, et al. Imaging features of idiopathic intracranial hypertension, including a new finding: widening of the foramen ovale. Acta Radiol 2012;53:682-88 CrossRef Medline

30. Topal O, Erbek SS, Erbek S, et al. Subjective pulsatile tinnitus associated with extensive pneumatization of temporal bone. Eur Arch Otorhinolaryngol 2008;265:123-25 Medline

31. Durgun B, Ilglt ET, Cizmeli MO, et al. Evaluation by angiography of the lateral dominance of the drainage of the dural venous sinuses. Surg Radiol Anat 1993;15:125-30 CrossRef Medline

32. Hofmann E, Behr R, Neumann-Haefelin T, et al. Pulsatile tinnitus: imaging and differential diagnosis. Dtsch Arztebl Int 2013;110: 451-58 CrossRef Medline

33. Carey JP, Minor LB, Nager GT. Dehiscence or thinning of bone overlying the superior semicircular canal in a temporal bone survey. Arch Otolaryngol Head Neck Surg 2000;126:137-47 CrossRef Medline

34. Mondina M, Bonnard D, Barreau X, et al. Anatomo-radiological study of the superior semicircular canal dehiscence of 37 cadaver temporal bones. Surg Radiol Anat 2013;35:55-59 CrossRef Medline 